\title{
Carrier localization and related photoluminescence in cubic AlGaN epilayers
}

\author{
R. E. L. Powell ${ }^{1}$, S. V. Novikov ${ }^{1}$, F. Luckert ${ }^{2}$, P. R. Edwards ${ }^{2}$, A. V. Akimov ${ }^{1,3}$, C. T. Foxon ${ }^{1}$, R. W. \\ Martin $^{2}$ and A. J. Kent ${ }^{1}$ \\ ${ }^{1}$ School of Physics and Astronomy, University of Nottingham, University Park, Nottingham NG7 2RD, UK. \\ ${ }^{2}$ Department of Physics, SUPA, University of Strathclyde, Glasgow, G4 ONG, UK \\ ${ }^{3}$ A.F.Ioffe Physical-Technical Institute, Russian Academy of Sceinces, St.Petersburg 194021, Russia
}

\begin{abstract}
The steady state and time-resolved photoluminescence (PL) spectra of cubic $\mathrm{Al}_{x} \mathrm{Ga}_{1-\mathrm{x}} \mathrm{N}$ have been measured for $0<x<1$. The intensity of the room temperature PL increases by an order of magnitude when the AIN content increases from $x=0$ to $x=0.95$. Additionally, the $\mathrm{PL}$ decay slows down with the decrease of temperature and increase of $x$. These results show that strong localization of carriers on alloy composition fluctuations plays a large role in determining the intensity and temporal evolution of the PL. The activation energy for the localized carriers increases with the increase of $x$ and reaches the value of $55 \mathrm{meV}$ at $x=0.95$.
\end{abstract}




\section{INTRODUCTION}

Semiconductor alloys are an integral part of electronic and optoelectronic devices. The variation of compound content in the alloy allows control of the band gap, $E_{g}$, of the semiconductor thus varying the transport and optical properties of the material and related heterostructures. One common feature of alloys is the existence of tails in the density of states and related carrier localization, which appear due to the statistical fluctuations of alloy composition. This decreases the carrier mobility, which in turn causes undesirable degradation of transport properties in electronic devices. On the other hand, the remarkable feature, which results from the localization phenomenon, is the increase of the near-band edge photoluminescence (PL) quantum efficiency, because photoexcited carriers and excitons, being localized, cannot easily reach the defects and surface states, where nonradiative processes can become dominant. In nitride compounds, like $(\mathrm{Al}, \mathrm{Ga}) \mathrm{N}$, large values of $E_{g}$ inevitably lead to a large scale of activation energies from the localized states. This promises the achievement of high PL efficiency at room temperature which makes possible the fabrication of efficient blue and ultraviolet LEDs and lasers based on nitrides, where, due to the technical difficulties, it is difficult to grow defect free material and exploit the free carrier and exciton radiative properties.

The effects of carrier localization and related PL have been studied in various semiconductor alloys over many years. For more detailed experimental and theoretical studies we refer readers to publications on (Al,Ga)As [1], II-VI compounds [2] and references therein. During the last decade there have also been a number of experiments on hexagonal AlGaN [3-7]. In some of these works, intense room temperature PL was observed due to localization of carriers on alloy fluctuations [4]. The latest achievements in growing cubic nitride epilayers with high stability show that these materials offer some potential advantages for fabrication of optoelectronic devices. The main advantages of cubic over hexagonal nitrides are the absence of the built in piezoelectric fields and the ability to use them in combination with other cubic III-V semiconductors, like GaAs. Thus it is attractive to study the possibility of exploiting the localization of carriers and excitons in cubic nitrides in order to achieve high quantum efficiency light emitting devices. To our knowledge, the localization of carriers in the alloys based on cubic nitride compounds has received very little attention to date.

In this work we present the results of PL studies for a series of $\mathrm{Al}_{\mathrm{x}} \mathrm{Ga}_{1-\mathrm{x}} \mathrm{N}$ samples with $\mathrm{x}=0-0.95$. The temperature dependencies of the PL intensity and its temporal decay clearly show the importance of localization effects in determining the carrier dynamics and respectively the PL spectra. The intensity at room temperature in the alloys with high $x>0.6$ is higher by two orders of magnitude in comparison with 
plain cubic (c-) GaN grown by the same technique. The samples with highest $x=0.95$ show the largest value for the localization energy of $55 \mathrm{meV}$.

The paper is organized in a following way: in Sec.ll the sample growing methods and the technique for PL measurements are described; in Sec.III the experimental results of the studies of PL spectra, their temperature dependence and time evolution are presented and the main arguments in support of carrier localization effects are given; finally, in Sec. IV, various aspects of carrier localization are discussed.

\section{EXPERIMENTAL DETAILS}

The zinc-blende (cubic) $\mathrm{Al}_{x} \mathrm{Ga}_{1-x} \mathrm{~N}$ films were grown on 2" diameter semi-insulating GaAs (001) substrates by plasma-assisted molecular beam epitaxy (PA-MBE) in a MOD-GENII system using arsenic $\left(\mathrm{As}_{2}\right)$ as a surfactant to initiate the growth of cubic phase material [8]. The active nitrogen for the growth of the group III-nitrides was provided by an HD25 RF activated plasma source. Prior to the growth of the (Al,Ga)N layers, a GaAs buffer layer was grown on the GaAs substrate in order to improve the properties of the cubic GaN layers. In the current study, all zinc-blende $\mathrm{Al}_{x} \mathrm{Ga}_{1-x} \mathrm{~N}$ layers were grown at temperatures $\sim 680^{\circ} \mathrm{C}$ at a growth rate of $\sim 0.25 \mu \mathrm{m} / \mathrm{hr}$. In order to sustain the cubic phase during the epitaxy it is crucial to maintain an excess of group III elements on the growth surface. We have used a new approach to maintain the excess of group III elements for the growth of $\mathrm{Al}_{x} \mathrm{Ga}_{1-x} \mathrm{~N}$ layers for a wide $\mathrm{Al}$ composition range: namely the use of an excess $\mathrm{Ga}$ flux [9]. Samples were studied in-situ using reflection high-energy electron diffraction (RHEED) and after growth ex-situ measurements were performed using X-ray diffraction (XRD). We have studied Al incorporation into cubic $\mathrm{Al}_{x} \mathrm{Ga}_{1-x} \mathrm{~N}$ layers by secondary ion mass spectrometry (SIMS) using Cameca IMS-3F and IMS-4F systems and by electron probe microanalysis (EPMA) using a Cameca SX100 apparatus. As a result of the measurements the AIN content $x$ was obtained with a fractional error of 1-2 percent.

The PL measurements were performed in a liquid helium flow cryostat with temperature stabilization in the range $5-300 \mathrm{~K}$. The PL was excited by deep-UV pulses from a quadrupled Ti-Sapphire laser. The photon energy of the excitation was $5.9 \mathrm{eV}$, duration of pulses $\sim 100 \mathrm{fs}$, repetition rate $82 \mathrm{MHz}$ and average power $2 \mathrm{~mW}$. The beam was focused to a spot with a diameter $\sim 50 \mu \mathrm{m}$. The time-integrated PL spectra were collected using parabolic reflective optics and analyzed by an Ocean Optics UV enhanced spectrometer. The time resolved measurements were performed using a photon counting system with a fast photomultiplier at the exit slit of a Bentham M300 monochromator and Becker and Hickl SPC-630 PC board with a sampling interval of $1.2 \mathrm{ps}$. The overall time resolution of the system was not worse than 150 ps. 


\section{EXPERIMENTAL RESULTS}

\section{A. Photoluminescence spectra}

Figure 1 shows the time integrated PL spectra for 10 samples with various contents of Al, measured at room temperature $T=300 \mathrm{~K}(\mathrm{a})$ and at $T=5 \mathrm{~K}$ (b). All spectra possess a peak in the ultraviolet spectral range which corresponds to the near band-edge PL of the corresponding alloy. The photon energy $E_{P L}{ }^{\max }$ corresponding to maximum intensity increases with the increase of $x$ which is due to the increase of $E_{g}$. The spectra measured at $\mathrm{T}=300 \mathrm{~K}$ show that $\mathrm{PL}$ intensity of this near band-edge $\mathrm{PL}$ rapidly increases with the increase of $x$. The integrated PL intensity for $x=0.95$ is two orders of magnitude higher than for the c-GaN layer $(x=0)$. Qualitatively this effect is similar to that observed earlier in hexagonal $(\mathrm{Al}, \mathrm{Ga}) \mathrm{N}$ and provides the first indication of carrier localization in the alloy with high $x$ [4]. The intensity increase of the PL with the increase of $x$ is also observed at $T=5 \mathrm{~K}$, but the difference for low and high $x$ is not as large as at room temperature.

The interesting feature of the low temperature PL spectra [Fig. 1(b)] is the modification of the spectral shape while $x$ increases: in c-GaN $(x=0)$ there are several narrow spectral lines, related to exciton annihilation, and a low energy sideband usually attributed to donor-acceptor recombination [10]. As $x$ is increased the low energy wing disappears and the sharp lines broaden. Finally the spectrum consists of one broad spectral peak or several overlapping peaks separated by much higher energy than the spectral distance between the exciton lines in the samples with low $x$. Such transformation of the low temperature spectra with the increase of $x$ is further qualitative evidence for carrier localization. Indeed, it is known that the localization on the alloy fluctuations results in the inhomogeneous broadening of the exciton PL lines and the spectra may consist of several broad peaks [1-7]. When the spectrum consists of more than one peak, a high energy one is usually attributed to the PL from the extended, i.e. band states, while more intense low energy peaks correspond to the PL from the localized states $[2,4]$.

The symbols in Fig. 2 show the $x$ dependence of the photon energy $E_{P L}{ }^{\max }$. The increase of $E_{P L}{ }^{\max }$ with the increase of $x$ is in qualitative agreement with the expansion of the band gap, $E_{g}$, obtained from the earlier work at $T=300 \mathrm{~K}$ [23]. However the values for $E_{g}$ and its direct or indirect origin in (Al,Ga)N alloys are still under the debate $[21,22,23]$. In analogy with hexagonal (AI,Ga)N [4] we may expect that the Stokes shift for the PL peak relative to $E_{g}$ at high values of $x$ is more than $100 \mathrm{meV}$ due to localization effects and electron-phonon interactions [2] in the studied samples.

\section{B. Temperature dependence of the PL spectra}


Figure 3 shows the series of PL spectra measured at various temperatures in samples with $x=0.23$ (a) and 0.95 (b). Strong temperature quenching is clearly observed in the sample with low AIN content [Fig. 3(a)]. Significantly weaker temperature quenching is also observed in the sample with high $x$ [Fig. 3(b)]. The modification of the spectral shape from relatively narrow peaks to a broad PL band, mentioned earlier, can be traced in the sample with lower $x$ [Fig. 3(a)]. In the sample with high $x=0.95$ [Fig. 3(b)] the spectral shape does not change significantly with $T$, but the shift of $E_{P L}{ }^{\max }$ by $\sim 50 \mathrm{meV}$ towards low energies is seen while $T$ increases from $5 \mathrm{~K}$ to $200 \mathrm{~K}$.

To be more specific we may estimate the activation energies $\Delta E$ from Arrhenius plots of the high temperature data [13]:

$$
I_{P L}=I_{0} /[1+C \exp (-\Delta E / k T)],
$$

where $I_{0}$ and $C$ are phenomenological parameters determined by the kinetics of electrons and holes and $\Delta E$ is the mean activation energy required for releasing the localized carriers into extended states where they have a high probability of recombining non-radiatively.

Examples of the $T$ dependencies of the PL peak intensity $I(T)$ measured at $E_{P L}=E_{P L}{ }^{\max }$ are shown in Fig. 4 for four samples. The best fits found using Eq.(1) are shown by solid lines. The dependence of $\Delta E(x)$ obtained from the fits of the $I(T)$ for various $x$ is shown in Fig.5. This dependence is an increasing function of $\mathrm{x}$. For high $\mathrm{x}, \Delta E$ essentially exceeds $k T$ even for the highest temperature ( $\mathrm{T}=300 \mathrm{~K}$ ) used in the experiments. This result supports the statement about the localization of carriers in the $\mathrm{Al}_{x} \mathrm{Ga}_{1-x} \mathrm{~N}$ alloys with high $\mathrm{x}$, even at room temperature. However, it should be noted that, as might be expected for alloy samples, it was not possible to describe the whole temperature range with a single value of the activation energy.

\section{Time resolved photoluminescence.}

Time resolved PL data are well known to provide valuable information about any localization effects [14]. If carriers which take part in the recombination are localized in the same microscopic area, as shown schematically in Fig. 6(a), then the decay time of PL from these electron-hole pairs (excitons) is governed by the recombination time $\tau_{R}$, which, for nonresonant excitation, has a value close to the regular alloy without fluctuations. At low $T$ for the nitrides and their alloys, $\tau_{R} \sim 10^{-10} \mathrm{~s}$ [15]. However, if the electron and hole are localized in areas separated in space [Fig.6 (b)], then the decay time may exceed $\tau_{R}$ by several orders of magnitude and it has a very strong dependence on the distance between the localized electron and hole. In general, due to a wide distribution of localization energies and distances between the localized 
electrons and holes, the PL decay $I(t)$ is non-exponential and depends on the alloy content, temperature, excitation density, excitation and detection wavelengths.

The PL decay curves $I(t)$ measured at $T=5 \mathrm{~K}$ for various AIN content are shown in Fig.7. It is seen that the decay is non-exponential and cannot be characterized by a single decay time. Such behavior, as already mentioned, is typical for the recombination of carriers localized on the alloy fluctuations. The linear part of the decay in the logarithmic plots immediately following the excitation pulse may be fitted by exponential decay with $\tau_{R} \sim 0.3-0.4 \mathrm{~ns}$ depending on $x$. At low temperature $I(t)$ does not differ strongly for various $x$. However, this is not a case for elevated temperatures. The decay speeds up and eventually reaches the limit of the temporal resolution while $T$ increases. Figures $8(a)$ and $(b)$ show $I(t)$ measured at various $T$ in two samples with different AIN content, $x=0.16$ and $x=0.49$ respectively. In the sample with low $x$ the temperature induced speed up of the decay starts at a lower $T$ than in the sample with high $x$. In our example (Fig. 8), $I(t)$ for $x=0.16$ (a) is barely distinguishable from the laser pulse already at $T=150 \mathrm{~K}$, while for $\mathrm{x}=0.49 \mathrm{~b}$ (b) $/(t)$ measured at $T=200 \mathrm{~K}$ still has a distinctly longer decay than the excitation pulse. Thus we may conclude that, at higher $x$, the carriers remain localized to higher temperatures.

Evidently the radiative recombination and thermal activation followed by nonradiative decay depends on the energy of the electrons, $E_{e}$, and holes, $E_{h}$, in the tails of the density of states. Hence, $I(t)$ for various emitted photon energies $E_{P L}=E_{e}-E_{h}$ should vary. As an example of the dependence of $I(t)$ on $E_{P L}$ we present data (Fig. 9) measured at $T=5 \mathrm{~K}$ for the sample with high AIN content ( $\mathrm{x}=0.78)$. It is seen that $I(t)$ decays faster as $E_{P L}$ moves from the low- to the high energy wing of the PL spectrum. Such behavior is typical for the PL from localized carriers. The electrons (holes) are more strongly localized at the bottom (top) of band tails and these strongly localized carriers have smaller overlap for radiative recombination [see Fig. 6(b)]. For lower $x$ and higher $T$ the dependence of the PL decay on $E_{P L}$ is governed additionally by thermal activation. This leads to the redistribution of carriers between various localized states and increases the probability for non-radiative decay. The detailed analysis of these dependencies, and also the excitation wavelength and power dependencies of the PL decay are the subject of future studies and lies beyond the scope of the present work.

\section{DISCUSSION AND CONCLUSIONS}

The main deduction which follows from the presented experimental results is the growing role of carrier localization in the PL with the increase of AIN content. To summarize the arguments in favor of this, we point out the following experimental observations which take place while $x$ increases: (i) the intensity of PL 
increases [see Fig. 1(a)]; (ii) the activation energy $\Delta E$ obtained from the Arrhenius plots increases (see Fig.5); and (iii) the decay of PL at elevated temperatures slows down (see Fig.8).

The presence of carrier localization and its important role in the PL of the studied cubic $(\mathrm{Al}, \mathrm{Ga}) \mathrm{N}$ alloys is not surprising and the general features, described in our work, have been observed in several types of III-V [1, 3-7] and II-VI [2] alloys. From the practical point of view, related to the applications of the cubic $(\mathrm{Al}, \mathrm{Ga}) \mathrm{N}$ alloys as a material for efficient LEDs and lasers, it is important to understand what values of AIN content in the alloy are most suitable for a particular structure or opto-electronic device. The value of $\Delta E$ defines the energy separation between extended carriers which possess high nonradiative rate $w_{N R}$, and localized states, where the carriers are likely to recombine radiatively with a rate $w_{R}$. The kinetic parameters $W_{N R}$ and $w_{R}$ depend on $x$ due to basic properties and specific technological procedures for growth of the cubic (Al,Ga)N alloys. The parameter $C$ in Eq. (1) is governed mostly by the ratio $w_{N R} / w_{R} \sim C$ and obviously plays a no less important role in the PL efficiency than the value of $\Delta E$. The presented experimental results provide the information needed to choose an optimum $x$ depending on the specific task. For instance, for achieving the highest PL efficiency of the material at room temperature one would choose $x>0.5$. When using $(\mathrm{Al}, \mathrm{Ga}) \mathrm{N}$ as the barriers in heterostructures, the high efficiency PL from the barriers becomes unfavorable and the optimum value of $x$ should be obtained based on the balance between localization, thermal activation and nonradiative recombination of carriers in the alloy.

In the qualitative analysis of the experimental data we did not consider the Coulomb interaction between electrons and holes and hence exciton formation. Such interaction is important when at least one type of the carriers (e.g. electrons) remains mobile and couples to another carrier (e.g. hole) forming an exciton with the binding energy between $E_{\mathrm{ex}}=25 \mathrm{meV}$ [16] and $75 \mathrm{meV}$ [17] depending on $x$. In the studied samples this can be true at low $x$ and low $T$ because the low temperature PL spectra consist of several narrow lines. However, at high $x$, when localization effects are strong enough, both types of carrier are localized on the fluctuations of the alloy. Indeed, the valence and conduction band offsets have similar values, for both GaN and AIN [18], and so the amplitude of the potential profile for electrons is expected to mirror the profile for holes. Thus the case when one type of carrier is localized and another remains free seems unlikely.

It is notable that the activation energy $\Delta E$ increases monotonically with the increase of $x$ [Fig.5]. No decrease of $\Delta E$ is observed when $\mathrm{x}$ becomes close to unity. The PL spectra do not to show features of the plain AIN, like spectral narrowing and exciton peaks. One of the reasons for such unusual behavior could be a certain fraction of clusters with hexagonal alloys in the material which appear during the growth due to stacking faults. The role of stacking faults in PL for the materials which show in parallel face centered cubic 
(fcc) and hexagonal lattices has been studied for many years $[19,20]$. The stacking faults result in the development of clusters with different lattice symmetry. The density and size of these clusters are a subject of more detailed study using X-ray micro-PL techniques. Qualitatively it is clear, that being higher in energy relative to cubic $(\mathrm{Al}, \mathrm{Ga}) \mathrm{N}$, the carriers from hexagonal clusters do not contribute to PL significantly, but play a certain role in the carrier kinetics governing the formation of a mobility edge for electrons. Thus the studied samples may possess larger values of $\Delta E$ in comparison with the pure cubic alloy. If the role of hexagonal clusters is really essential, then even in pure AIN $(x=1)$, the carriers will be still strongly localized due to the presence of hexagonal AIN clusters. This is an opposite case to pure hexagonal AIN where PL spectra show narrow exciton lines and no features of localization effects are observed [21]. Another possibility is that the growth under excess Ga flux results in strong lateral alloy fluctuations about the mean composition, even for large $x$ samples, as discussed in Ref. [24]

In conclusion, we have shown that photoexcited electrons and holes in cubic AlGaN alloys at high $x>0.5$ possess strong localization at room temperature. As a result, the intensity of the near-band edge PL increases by more than 2 orders of magnitude in comparison with pure cubic GaN. The activation energy for localized carriers increases linearly with AIN content and at $x=0.95$ it reaches the value of $55 \mathrm{meV}$. The decay of PL from localized carriers is nonexponential due to the spatial separation of electrons and holes.

The authors greatfully acknowledge Prof. Alexander Reznitsky for useful discussion and EPSRC for financial support.

\section{References}

1. L. Pavesi, and M. Guui, J. Appl. Phys. 75, 4779 (1994).

2. A. Klochikhin, A. Reznitsky, S. Permogorov, T. Breitkopf, M. Gru“n, M. Hetterich, C. Klingshirn, V. Lyssenko, W. Langbein and J. M. Hvam, Phys. Rev. B 59, 12947 (1999).

3. J. Li, K. B. Nam, J. Y. Lin, and H. X. Jiang, Appl. Phys. Lett. 79, 3245 (2001).

4. C. J. Collins,a! A. V. Sampath, G. A. Garrett, W. L. Sarney, H. Shen, M. Wraback, A. Yu. Nikiforov, G. S. Cargill,and V. Dierolf, Appl. Phys. Lett. 86, 031916 (2005).

5. N. Nepal, J. Li, M. L. Nakarmi, J. Y. Lin, and H. X. Jiang, Appl. Phys. Lett. 88, 062103 (2006).

6. E. Kuokstis, W. H. Sun, M. Shatalov, J. W. Yang, and M. Asif Khan, Appl. Phys. Lett. 88, 261905 (2006).

7. K. B. Lee, P. J. Parbrook,T. Wang, F. Ranalli, T. Martin, R. S. Balmer, and D. J. Wallis, J. Appl. Phys. 101, 053513 (2007).

8. S.V. Novikov, N.M. Stanton, R.P. Campion, C.T. Foxon, and A.J. Kent, J. Cryst. Growth 310, 3964 (2008).

9. S. V. Novikov, C. R. Staddon, C. T. Foxon, F. Luckert, P. R. Edwards, R. W. Martin and A. J. Kent, J. Cryst. Growth, accepted.

10. K. Lischka, Brazilian J. of Physics 27/A, 80 (1997). 
11. M. Grundmann, and C.P. Dietrich, J. Appl. Phys. 106, 123521 (2009).

12. M. Röppischer, R. Goldhahn, G. Rossbach, P. Schley, C. Cobet, N. Esser, T. Schupp, K. Lischka, and D. J. As, J. Appl. Phys. 106, 076104 (2009).

13. J.I. Pankove, Optical Processes in Semiconductors, (Dover, New York, 1975), p.166.

14. S. A. Permogorov, and A. N. Reznitsky, J. Lumin., 52, 201 (1992).

15. O. Brandt, J. Ringling, K. H. Ploog, H.-J. Wunsche and F. Henneberger, Phys. Rev. B 58, R15977 (1998).

16. S. J. Xua, W. Liu and M. F. Li, Appl. Phys. Lett. 81, 2959 (2002).

17. L. Roskovcova, and J. Pastrnak, Czech. J. Phys. B 30586 (1980).

18. J.R. Waldrop, and R.W. Grant, Appl. Phys. Lett. 68, 2879 (1996).

19. A. I. Ryskin, L. G. Suslina, G. I. Khilko, and E. B. Shadrin, Phys. Stat. Solid (b) 49875 (1972).

20. E. J. Tarsa, B. Heying, X. N. Wu, P. Fini, S. P. DenBaars, and J. S. Speck, J. Appl. Phys. 82, 5472 (1997).

21. A. Sedhain, N. Nepal, M. L. Nakarmi, T. M. Al tahtamouni, J. Y. Lin, H. X. Jiang, Z. Gu, and J. H. Edgar, Appl. Phys. Lett. 93, 041905 (2008).

22. M. Marques, L. K. Teles, L.M.R. Scolfaro, J.R. Leite, J. Furthmuller and F. Bechstedt, Appl. Phys. Lett. 83, 890 (2003).

23. T. Suzuki, H. Yaguchi, H. Okumura, Y. Ishida and S. Yoshida, Jpn. J. Appl. Phys. 39, L497 (2000).

24. Y. Liao, C. Thomidis, C-K. Kao and T. D. Moustakas, Appl. Phys. Lett. 98, 081110 (2011). 
Figure captions.

Fig. 1 PL spectral measured for various AIN content, $x$, at room temperature (a) and at $T=5 \mathrm{~K}$ (b). The intensity of PL can be traced in (a) while in (b) the intensities are normalized to maximum.

Fig.2. The dependence of the photon energy at the maximum of the PL intensity on the AIN content at room temperature (squares) and $\mathrm{T}=5 \mathrm{~K}$ (triangles).

Fig.3. PL spectra measured at various temperatures for the samples with $x=0.23$ (a) and $x=0.95$ (b).

Fig.4. PL intensity for the samples with various AIN content as a function of inverse temperature. Corresponding Arrhenius plots are shown by solid lines.

Fig.5. Activation energy for localized carriers as a function of AIN content.

Fig.6. The schematic diagram of radiative transitions when the electron and hole are localized in the same place (a) and separated in space (b).

Fig.7. The temporal evolution of PL for the samples with various AIN content measured at $T=5 \mathrm{~K}$. The dashed and dashed-dotted lines show the exponential decay with time constants 300 ps and 280 ps respectively.

Fig.8. The temporal evolutions of PL measured at various temperatures in two samples with AIN content $x=0.16(a)$ and $x=0.49(b)$.

Fig.9. The temporal evolution of PL measured for sample with $\mathrm{x}=0.78$ at $\mathrm{T}=5 \mathrm{~K}$ and various photon energies $E_{P L}$ indicated by the arrows in the inset which shows the PL spectrum. 

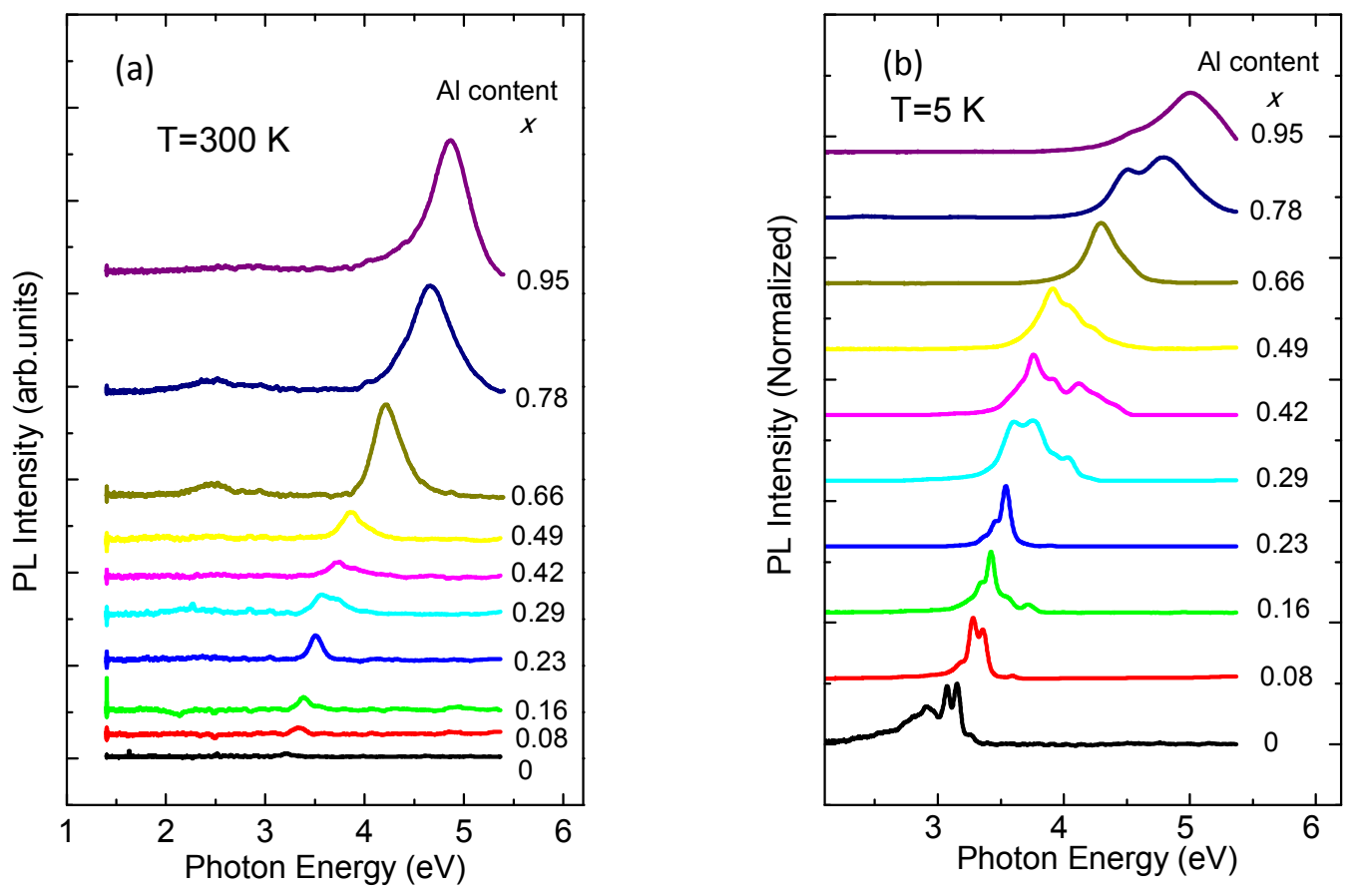

Figure 1 


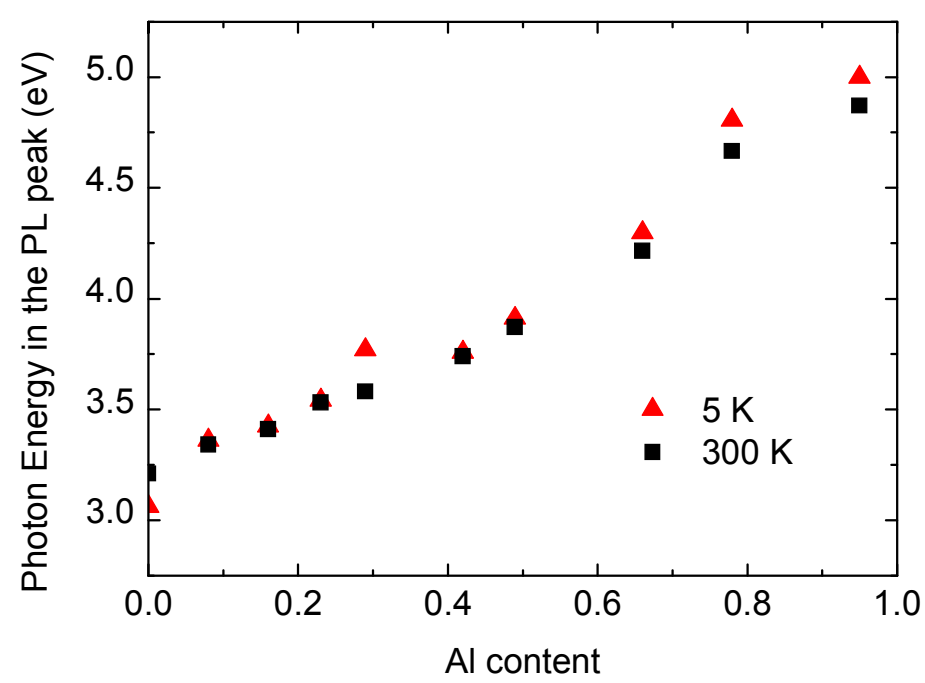

Figure 2 

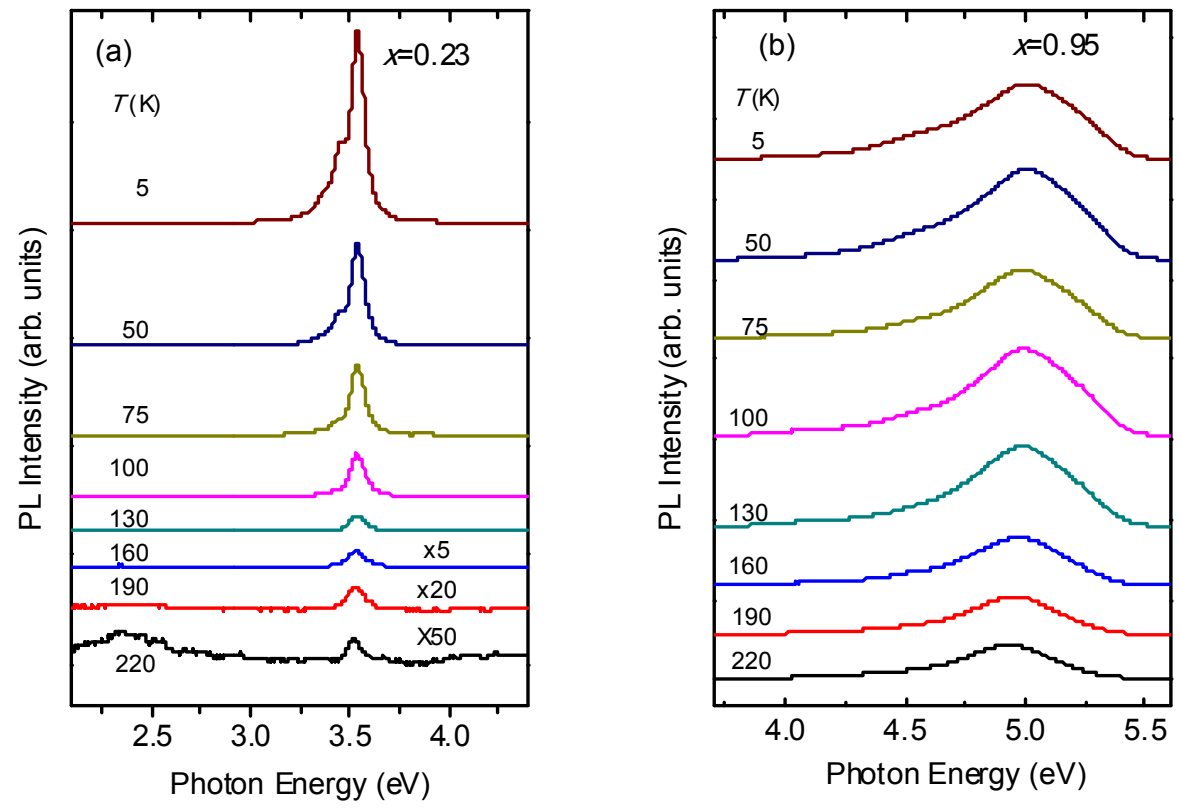

Figure 3 


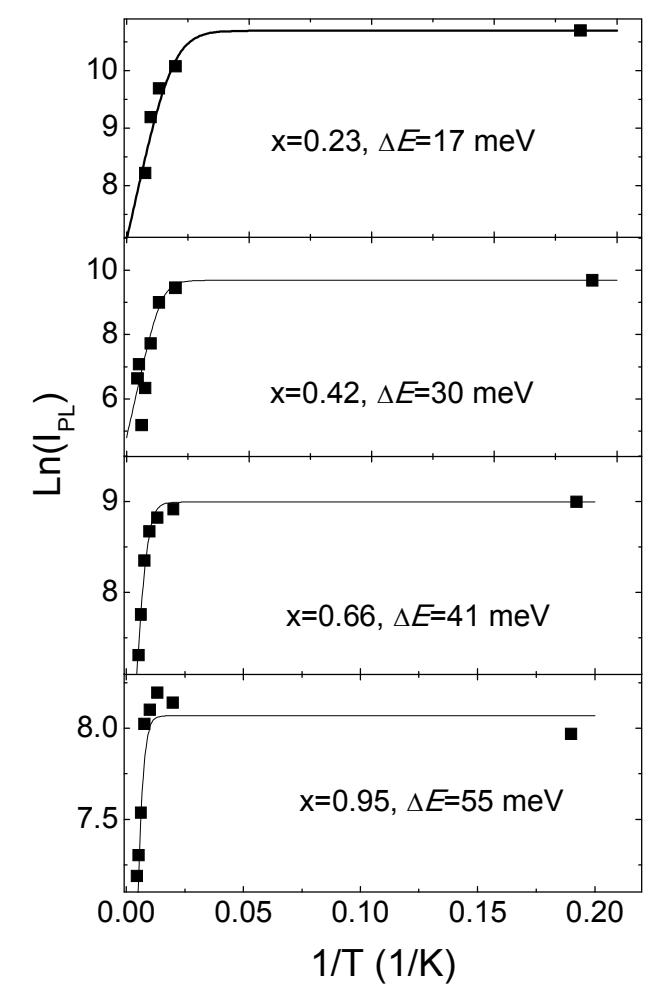

Figure 4 


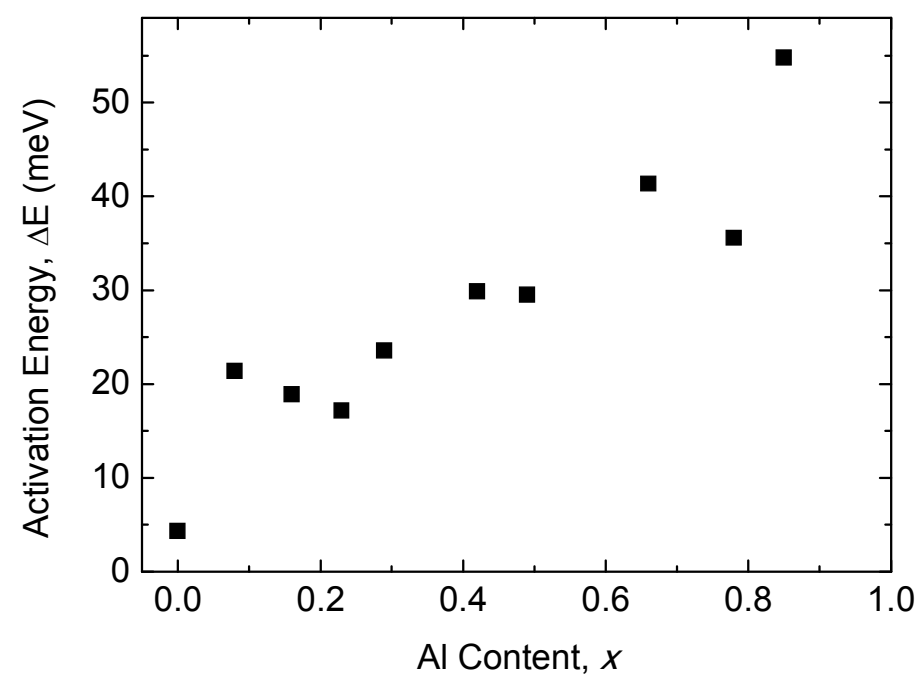

Figure 5 
(a)

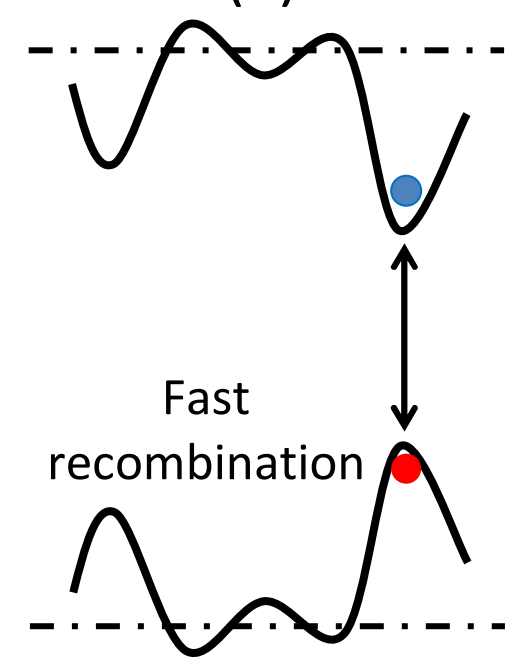

(b)

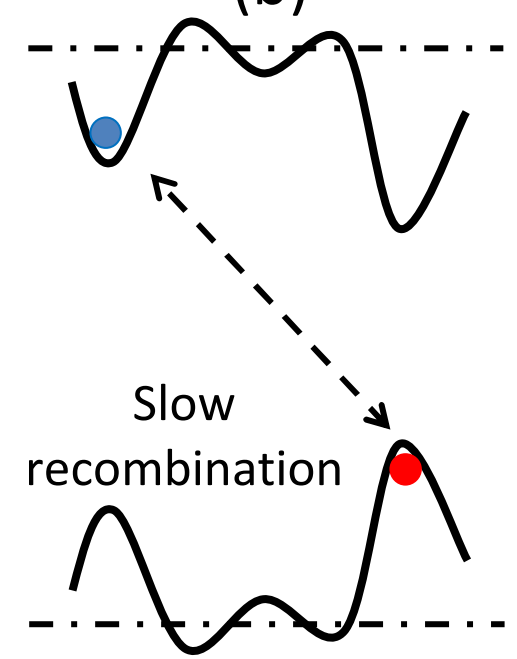

Figure 6 


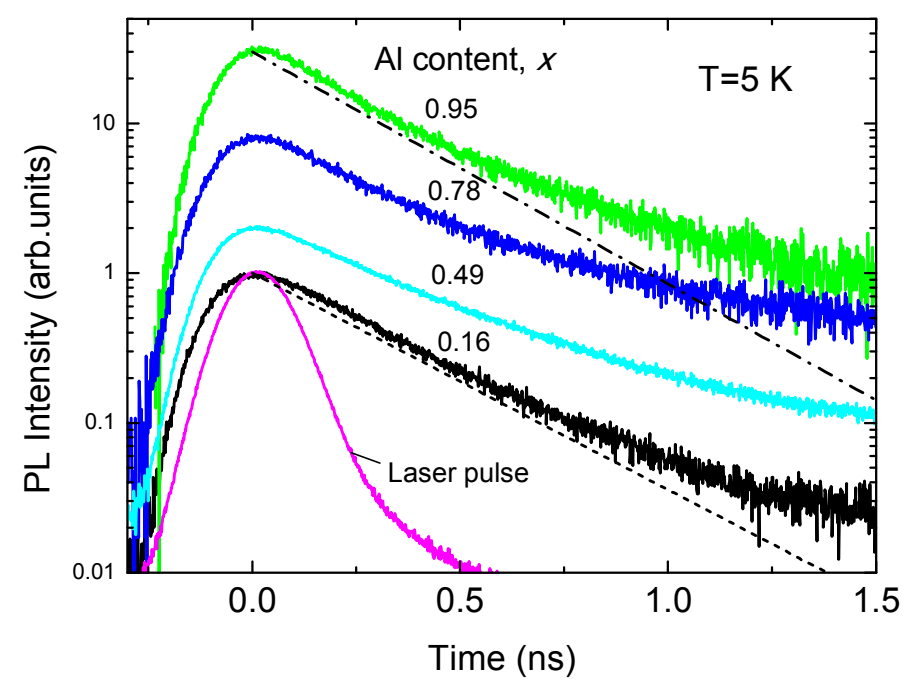

Figure 7 

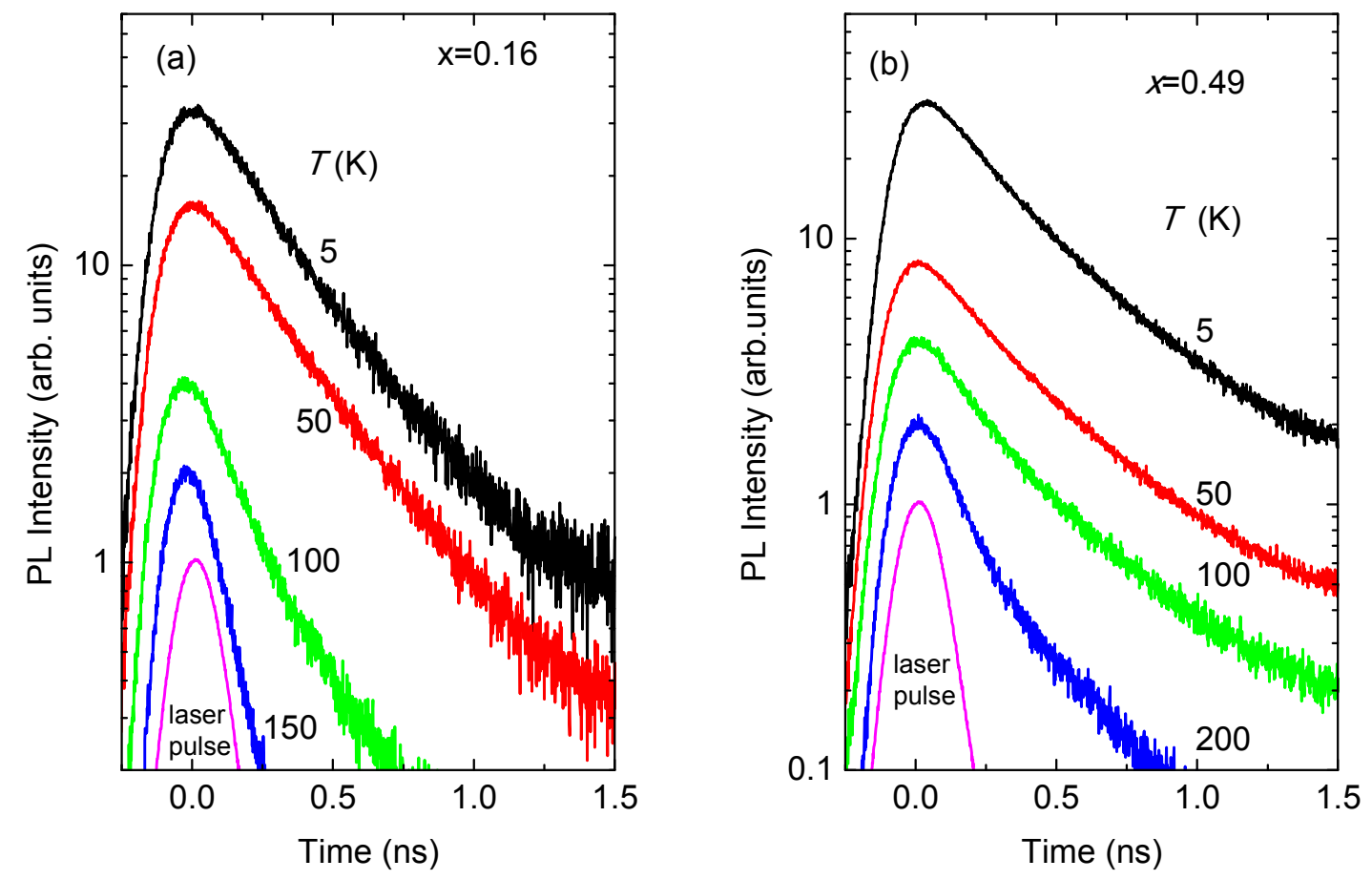

Figure 8 


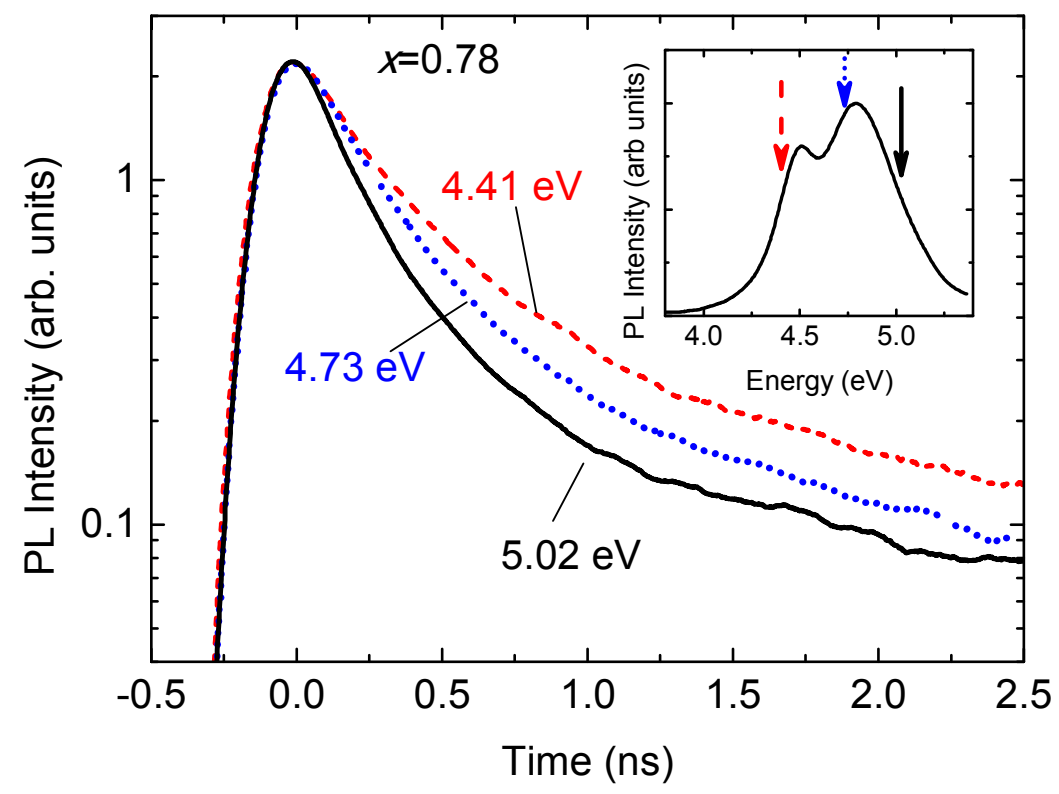

Figure 9 